\title{
On the Use of Positive Polynomials for the Estimation of Upper and Lower Expectations in Orbital Dynamics
}

\author{
Massimiliano Vasile and Chiara Tardioli
}

\begin{abstract}
The paper presents the use of positive polynomials, in particular Bernstein polynomials, to represents families of probability distributions in orbital dynamics. The uncertainty in model parameters and initial conditions is modeled with p-boxes to account for imprecision and lack of knowledge. The resulting uncertainty in the quantity of interest is estimated by representing the upper and lower expectations with positive polynomials with interval coefficients. The impact probability of an asteroid subject to a partially known Yarkovsky effect is used as an illustrative example.
\end{abstract}

\section{Introduction}

The treatment of uncertainty in orbit propagation is of fundamental importance to predict the motion of natural and man-made objects. In the specific case of asteroids and space debris a key quantity of interest is the probability of an impact with the Earth or a collision with an operational satellite.

Several methods have been proposed to deal with uncertainty and provide a prediction of the future state of a space object. Most of them start from some assumptions on the probability distribution associated to the uncertain quantities and then model, more or less accurately, the distribution of the quantity of interest. When the nature of uncertainty is epistemic (lack of knowledge), a single probability distribution might not be available. More likely different sources of information may suggest that the probability associated to an uncertain quantity belongs to a finite set for which we can define upper and lower bounds.

However, the fast calculation of these bounds is not a trivial matter. In this paper an approach based on the use of positive polynomials is proposed to calculate

Massimiliano Vasile, Chiara Tardioli

Aerospace Centre of Excellence, University of Strathclyde, 75 Montrose Street, G11XJ, Glasgow, UK, e-mail: [massimiliano.vasile - chiara.tardioli]@strath.ac.uk 
the upper and lower bounds via a simple linear optimisation programme. The uncertain quantities are modeled with p-boxes defined through parametric probability distributions or via positive polynomial expansions [4].

The calculation of the impact probability of an asteroid subject to a poorly known Yarkovsky effect is used as an illustrative example.

\section{Worst Case Scenario}

The problem under investigation is to evaluate the probability of the following set of events:

$$
A_{v}=\left\{\mathbf{u} \in \mathfrak{U}_{0}: f(\mathbf{u}) \leq v\right\}
$$

where $f$ is the quantity of interest and $\mathbf{u}$ is a stochastic variable defined in an uncertainty space $\mathfrak{U}_{0}$ with dimension $d$. We use the notation $[\mathbf{u}] \in \mathbb{R}^{d}$ to indicate the convex set of $\mathbf{u}$ such that $\mathbf{u} \in \mathfrak{U}_{0} \subseteq \mathbb{R}^{d}$. If $d=1$, the uncertainty is an interval and it is also indicated as $[\underline{u}, \bar{u}]$, where $\underline{u}, \bar{u}$ are the lower and upper limit, respectively.

Regardless of the distribution of $\mathbf{u}$ one can define the best and worst case scenarios as follows:

$$
\underline{f}=\min _{\mathbf{u} \in \mathfrak{U}_{0}} f(\mathbf{u}), \quad \bar{f}=\max _{\mathbf{u} \in \mathfrak{U}_{0}} f(\mathbf{u}) .
$$

The solution to (20) gives the limit of variability of $f$ and identifies also two rare events. For any value of $v \in[\underline{f}, \bar{f}]$ and a known probability distribution $p$, the probability associated to $A_{v}$ is given by the formula

$$
I P\left(A_{v}\right)=\int_{A_{v}} p(\mathbf{u}) \mathrm{d} \mathbf{u}
$$

In the following, the uncertain variables are assumed to be independent and uncorrelated so that the initial uncertainty space is the hyper-rectangle, however, the solution of Eqs. (20) does not require $\mathfrak{U}_{0}$ to be a box and holds true for any generic set. The same is true for Eq.(22).

\section{Upper and Lower Expectations}

When the uncertainty on the input quantities is epistemic the probability $p$ can belong to a family of parametric distributions or to a set of unknown distributions. 


\subsection{Representation with Families of Parametric Distributions}

Consider the case in which one can reasonably assume that the uncertainty can be quantified with a family of beta distributions with unknown parameters $\alpha$ and $\beta$ (any other parametric or non-parametric distribution would equally work). Eq. (22) then translates into two equations defining the upper and lower probability associated to $A_{V}$ :

$$
\min _{\alpha, \beta} \int_{A_{v}} p(\mathbf{u}) d \mathbf{u}, \quad \max _{\alpha, \beta} \int_{A_{v}} p(\mathbf{u}) d \mathbf{u},
$$

where $p$ is the product of probability $p=\prod_{j=1}^{d} p_{j}$, where each marginal density mass $p_{j}$ is a beta distribution function with parameters $\alpha_{j}, \beta_{j}$.

\subsection{Representation with Positive Polynomials}

In the general case the integrals in Eqs.(4) can calculated numerically via multidimensional quadrature formula. As an example we can replace the calculation of the exact integrals with an approximation using Halton low discrepancy sequence to generate $M$ sample points (called quasi-Monte Carlo points) in the domain $\mathfrak{U}_{0}$ and then re-write the integrals in the form:

$$
\int_{A_{V}} p(\mathbf{u}) d \mathbf{u} \approx \frac{1}{M} \sum_{k=1}^{M} I_{A_{v}}\left(\mathbf{u}_{k}\right) p\left(\mathbf{u}_{k}\right)
$$

where the samples $\mathbf{u}_{k}$ are taken from the low discrepancy sequence. Similarly, we can approximate the integrals in Eq. (4):

$$
\min _{\alpha, \beta} \sum_{k=1}^{M} I_{A_{v}}\left(\mathbf{u}_{k}\right) \prod_{j} p_{j}\left(\mathbf{u}_{k}\right), \quad \max _{\alpha, \beta} \sum_{k=1}^{M} I_{A_{v}}\left(\mathbf{u}_{k}\right) \prod_{j} p_{j}\left(\mathbf{u}_{k}\right) .
$$

subject to the constraint:

$$
\frac{1}{M} \sum_{k=1}^{M} p\left(\mathbf{u}_{k}\right)=1
$$

If the family of distributions is unknown or does not contain only one particular type, one can use an a representation with an expansion in positive polynomials to approximate the extrema of $[p]$ and obtain the upper and lower expectation on $A_{V}$ as solutions of a linear problem. In this paper, in particular, we propose the use of Bernstein polynomials [4][7]. The family of probability distributions to which the uncertain variable $u_{j}$ belongs can be expressed as

$$
\left[p_{c_{j}}\right]=\left\{\sum_{i=1}^{n} c_{i}^{(j)} B_{i}\left(\tau_{j}\left(u_{j}\right)\right)\right\}
$$


where $B_{i}:[0,1] \mapsto[0,1]$ is the $i^{t h}$-univariate Bernstein polynomials of dimension $n$ and $\tau_{j}$ is the change of coordinate from the uncertain interval $\left[u_{j}\right]$ to $[0,1]$.

Under the independence and non-correlation assumption among the variables, the joint probability distribution is the product of the marginal masses and it is contained in the p-box $\left[p_{\tilde{c}}\right]=\prod_{j=1}^{d}\left[p_{c_{j}}\right]$ which can be re-written as

$$
\left[p_{c}\right]=\left\{\sum_{\kappa \in \mathscr{K}} c_{\kappa} \mathscr{B}_{\kappa}(\tau(\mathbf{u}))\right\}
$$

with $\mathscr{K}=\left\{\kappa=\left(k_{1}, \ldots, k_{d}\right) \in \mathbb{N}^{d}: 0 \leq k_{j} \leq n, \forall j\right\}, \mathscr{B}_{\kappa}$ is a multivariate Bernstein polynomial, $\tau=\prod_{j=1}^{d} \tau_{j}$, and $c$ is the unknown coefficient vector. Then, the upper and lower expectation are the solutions of the two linear optimization problems:

$$
E_{l}\left(A_{V}\right)=\min _{c \in \mathscr{C}} \int_{A_{v}} p_{c}(\mathbf{u}) \mathrm{d} \mathbf{u}, \quad E_{u}\left(A_{v}\right)=\max _{c \in \mathscr{C}} \int_{A_{v}} p_{c}(\mathbf{u}) \mathrm{d} \mathbf{u},
$$

The set $\mathscr{C} \in \mathbb{R}^{M}$ can be assumed to be an hyper-cube, for example, $\mathscr{C}=[0, M]^{M}$. In discrete form programmes (10) translate into:

$$
E_{l}\left(A_{V}\right)=\min _{c \in \mathscr{C}} \sum_{s=1}^{M} I_{A_{v}}\left(\mathbf{u}_{s}\right) \sum_{\kappa \in \mathscr{K}} c_{\kappa} \mathscr{B}_{K}\left(\tau\left(\mathbf{u}_{s}\right)\right)
$$

and

$$
E_{u}\left(A_{v}\right)=\max _{c \in \mathscr{C}} \sum_{s=1}^{M} I_{A_{v}}\left(\mathbf{u}_{s}\right) \sum_{\kappa \in \mathscr{K}} c_{\kappa} \mathscr{B}_{\kappa}\left(\tau\left(\mathbf{u}_{s}\right)\right) .
$$

subject to the linear constraint:

$$
\frac{1}{M} \sum_{s=1}^{M} \sum_{\kappa \in \mathscr{K}} c_{\kappa} \mathscr{B}_{\kappa}\left(\tau\left(\mathbf{u}_{s}\right)\right)=1
$$

\subsection{Impact probability}

Positive polynomials are here applied to the estimation of upper and lower impact probabilities of an asteroid subject to the Yarkovsky effect.

We consider a simplified dynamical model of an asteroid under the gravitational force of the Sun and of the Yarkovsky effect. The latter is assumed to be a purely transverse acceleration $A_{2} / r^{2}$, where $r$ is the heliocentric distance and $A_{2}$ is a function of the asteroid physical quantities[3]. The dynamical equations, expressed in Keplerian orbital elements, can be reduced to

$$
\frac{d a}{d t}=\frac{2 A_{2}\left(1-e^{2}\right)}{n p^{2}}, \quad \frac{d M}{d t}=n,
$$


where $e$ is the eccentricity, $M$ is the mean anomaly, $n=\sqrt{\mu / a^{3}}$ is the mean motion of the unperturbed orbit with $\mu$ the gravitational parameter, and $p=a\left(1-e^{2}\right)$ is the conic parameter. For $A_{2}=0$ the dynamics (14) reduces to a pure Keplerian motion, while the semi-major axis drifts outwards for $A_{2}>0$, and inwards for $A_{2}<0$.

Although $A_{2}$ is unknown, it can be estimated using the available information on the physical model. Following Farnocchia et al.[3], the coefficient $A_{2}$ is expressed as

$$
A_{2}=\frac{4(1-A)}{9} \Phi(1 \mathrm{au}) f(\Theta) \cos \gamma, \quad f(\Theta)=\frac{0.5 \Theta}{1+\Theta+0.5 \Theta^{2}},
$$

where $\Phi(1 \mathrm{au})$ is the standard radiation force factor at 1 astronomical unit, A is the Bond albedo, $\Theta$ is the thermal parameter, and $\gamma$ is the obliquity. The radiation force at $1 \mathrm{~W} / \mathrm{m}^{2}$ is computed as

$$
\Phi(r)=\frac{3 L_{0}}{2 c \rho D},
$$

where $L_{0}$ is the luminosity of the Sun, i.e., the total power output of the source, $R$ is the mean radius of the asteroid, $m_{a}$ the mass of the asteroid, and $c$ is the velocity of light.

Using Bowel et al.[1], the Bond albedo can be written as $A=(0.29+0.684 G) p_{\mathrm{v}}$, with $G$ the slope parameter and $p_{\mathrm{v}}$ the geometric albedo. Farnocchia et al.[3] related the thermal parameter $\Theta$ to the thermal inertia $\Gamma$ :

$$
\Theta=\frac{\Gamma}{\varepsilon \sigma T_{s s}^{3}} \sqrt{\frac{2 \pi}{P_{r o t}}},
$$

where $\varepsilon$ is the emissivity coefficient, $\sigma$ is the Stefan-Boltzmann constant, $P_{r o t}$ is the rotation period, and $T_{s S}$ is the subsolar temperature[2]

$$
T_{s s}=\left[\frac{(1-A) L_{0}}{\eta \varepsilon \sigma r^{2}}\right]^{1 / 4}
$$

where $r$ is the heliocentric distance of the body and $\eta$ is the so-called beaming parameter, which is equal to one in the case that each point of the surface is in instantaneous thermal equilibrium with solar radiation.

Delbò et al.[2] related the thermal inertia to the diameter $D$ (in $\mathrm{km}$ ) by the expression

$$
\Gamma=d_{0} D^{-\psi},
$$

with $d_{0}=300 \pm 45 \mathrm{Jm}^{-2} \mathrm{~s}^{-1 / 2} \mathrm{~K}^{-1}$ and $\psi=0.36 \pm 0.09$.

Eventually, the diameter can be related to the absolute magnitude $H$ and the geometric albedo by the formula[6]

$$
D=1329 \frac{10^{-H / 5}}{\sqrt{p_{\mathrm{v}}}} .
$$

The main uncertainty is represented by the obliquity angle: according to La Spina et al.[5] retrograde and direct rotators are in a 2:1 ratio with the NEO population. 
Therefore, both the inward and the outward drift of the semi-major axis are possible. In addition, other key physical parameters are known with uncertainty. Due to the lack of knowledge in their distributions, they need to be treated as epistemic uncertainty variables.

It is assumed that both the initial conditions and the model parameters are uncertain. The uncertainty space is $\mathfrak{U}_{0}=\left[\mathbf{x}_{0}\right] \times[\mathbf{q}] \subset \mathbb{R}^{10}$, where $\mathbf{x}_{0}=\left(a_{0}, e_{0}, I_{0}, \Omega_{0}, \omega_{0}, \ell_{0}\right)$ is the initial Keplerian orbital element vector, and $\mathbf{q}=\left(D, G, p_{\mathrm{v}}, \rho, d_{0}, \psi, P_{r o t}, \gamma\right)$ is the model parameter vector.

The impact risk is computed at the close approach epoch using the projection on the target plane and the impact parameter $b$. We say that a collision may occur if the b-parameter is less or equal a safety radius: $b \leq R^{*}$; this threshold is fixed here at 1.5 Earth radii.

Due to the uncertainty in the initial conditions, the final states of the asteroid defined a connected region more or less elongated along its orbit. Therefore, we say that the significant uncertainty of the b-parameter is contained in the interval $[\underline{b}, \bar{b}] \subseteq \mathbb{R}$ given by

$$
\underline{b}=\min _{\mathbf{u} \in \mathfrak{U}_{0}} b(\mathbf{u}), \quad \bar{b}=\max _{\mathbf{u} \in \mathfrak{U}_{0}} b(\mathbf{u}) .
$$

Assuming that the orbital elements are uncertain with known distributions (aleatory uncertainty), while the model parameters are uncertain with unknown distributions (epistemic uncertainty), the product of their probabilities is epistemic and it is indicated with the probability box (shortly, p-box) $[p]$. Then the upper and lower impact probabilities is given by the formula

$$
\begin{aligned}
& P_{u}\left(A_{R^{*}}\right)=\max _{p \in[p]} \int_{A_{R^{*}}} p(\mathbf{u}) \mathrm{d} \mathbf{u}, \\
& P_{l}\left(A_{R^{*}}\right)=\min _{p \in[p]} \int_{A_{R^{*}}} p(\mathbf{u}) \mathrm{d} \mathbf{u},
\end{aligned}
$$

where $A_{R^{*}}=\left\{\mathbf{u} \in \mathfrak{U}_{0}: b(\mathbf{u}) \leq R^{*}\right\}$ is the event of interest.

We can now assume that each uncertainty variables is contained in a probability box (p-box) delimited by two Beta distribution functions:

$$
\left[p_{i}\right]=\left\{\operatorname{cdf}_{\operatorname{Beta}(\alpha, \beta)}: 1 \leq \alpha, \beta \leq 3\right\},
$$

where $i$ is the variable index. This is the situation in which there are two experts with opposite opinions: one believes that the most probable value is the left extrema of the interval $(\operatorname{Beta}(1,3))$ and the other that most probable value is the right extrema of the interval $(\operatorname{Beta}(3,1))$; and in the uncertainty analysis we want to take into account both of them. Each p-box can be re-defined as in Eq. (9) with $\mathscr{B}_{k}, k=1, \ldots, M$ multivariate Bernstein polynomials of degree 2. This is due to the fact that each Beta function can be approximated with a positive polynomials series. The degree 2 is because $\operatorname{Beta}(3,1)$, Beta $(3,1)$ are approximated exactly with a Bernstein polynomial of order 2 . 
Figure 1 shows the cumulative distribution function of the b-parameter corresponding to an aleatory and epistemic case. Curve $P$ represents the case when all variables are aleatory with known Beta distributions with parameters $\alpha=3, \beta=3$ for the orbital elements and Beta functions $\alpha=1, \beta=1$ (uniform distribution) for the Yarkovsky parameters. On the contrary when uncertainty on the model parameters and initial conditions is epistemic one obtains the upper and lower expectations (curves $E_{u}$ and $E_{l}$, respectively). For all the possible values of the uncertain parameters the impact probability in Eq. (22) is 1 since $b \leq 1.5 R_{\oplus}$, with $R_{\oplus}$ the Earth radius, for every $b$.

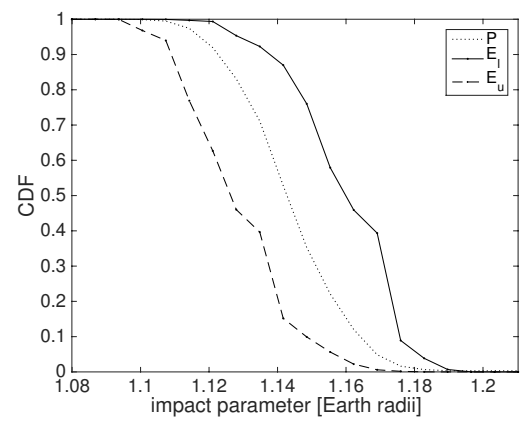

Fig. 1: Impact probability before deflection

From the same analysis one can estimate an upper and lower expectation of the Yarkovsky parameter $A_{2}$. Following Section 3.2, we can solve Eq. (10) with $\mathscr{C}=$ $[0, M]^{M}, M=6,561$, and the integral approximation given by Eq. (6) on $2 \cdot 10^{5}$ quasiMonte Carlo samples. The upper and lower expectation delimiting the p-box are computed on 50 bins in the interval $[-524,524] \mathrm{au} / \mathrm{d}^{2}$, using Bernstein polynomials as described in Section 3.2 on $10^{4}$ quansi-Monte Carlo points. The p-box of $A_{2}$ is shown in Figure 2. The Yarkovsky parameter is computed at a fixed distance of $a \sqrt{1-e^{2}}$. In the dynamical model we will sample $A_{2}$ from distributions $P$ such that $E_{l} \leq P \leq E_{u}$. To simplify the problem the upper and lower expectation have been approximated by Beta functions (Figure 2):

$$
E_{u} \approx \operatorname{cdf}_{\operatorname{Beta}(5,10)} \quad E_{l} \approx \operatorname{cdf}_{\operatorname{Beta}(10,5)}
$$

\section{Conclusion}

The paper demonstrates the use of positive polynomial expansions to approximate upper and lower expectations on the impact probability of an asteroid with the Earth. The proposed approach leads to the solution of a simple linear programme with a 


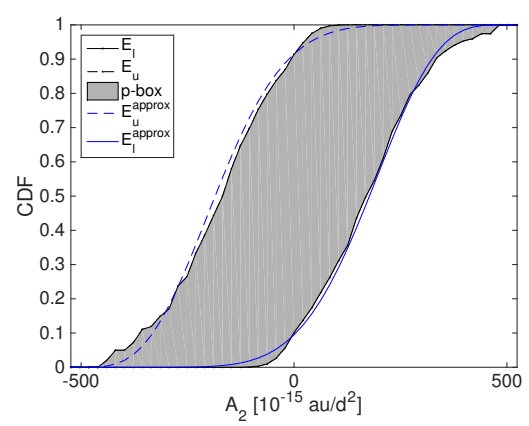

Fig. 2: Upper and lower expectation of the Yarkovsky parameter $A_{2}$

single linear constraint. The use of Bernstein polynomials, as proposed in this paper, allows for the representation of any set of probability distributions with finite support. The main limitation is the exponential growth of the number of polynomial coefficients with the number of dimensions. However, this problem is equally present in Gaussian mixture models although in this case no parameters, appearing nonlinear in the mixture model, need to be defined. The number of terms in the expansion can be calibrated to achieve the desired representation. Furthermore, the approach in this paper can be applied in conjunction with an high-dimensional representation of the quantity of interest that would mitigate the curse of dimensionality.

\section{Acknowledgment}

The work in this paper was partially supported by the Marie Curie FP7-PEOPLE2012-ITN Stardust, grant agreement 317185.

\section{References}

1. Bowell, E., Hapke, B., Domingue, D., Lumme, K., Peltoniemi, J., Harris, A.W.: Application of photometric models to asteroids. In: R.P. Binzel, T. Gehrels, M.S. Matthews (eds.) Asteroids II, pp. 524-556 (1989)

2. Delbò, M., dell'Oro, A., Harris, A.W., Mottola, S., Mueller, M.: Thermal inertia of near-Earth asteroids and implications for the magnitude of the Yarkovsky effect. Icarus 190, 236-249 (2007). DOI 10.1016/j.icarus.2007.03.007

3. Farnocchia, D., Chesley, S., Vokrouhlický, D., Milani, A., Spoto, F., Bottke, W.: Near earth asteroids with measurable yarkovsky effect. Icarus 224, 1-13 (2013)

4. Ghosal, S.: Convergence rates for density estimation with bernstein polynomials. Annals of Statistics 10(3), 1264-1280 (2001)

5. La Spina, A., Paolicchi, P., Kryszczynska, A., Pravec, P.: Retrograde spins of near-earth asteroids from the yarkovsky effect. Nature 428, 400-401 (2004) 
6. Pravec, P., Harris, A.W.: Binary asteroid population. 1. angular momentum content. Icarus 190, 250-259 (2007)

7. Zhao, Y., Ausn, C., Wiper, M.P.: Bayesian multivariate bernstein polynomial density estimation. In: UC3M Working papers. Statistics and Econometrics. Universidad Carlos III de Madrid. Departamento de Estadstica (2013) 